\title{
An Impact of Simulated Interprofessional Workshop on Healthcare Professionals' and Patients' Values Recognition
}

\author{
Desak Ketut Ernawati ${ }^{*}$, I Gusti Putu Suka Aryana2, Hirotaka Onishi ${ }^{3}$ \\ ${ }^{1}$ Department of Pharmacology and Therapeutics, Faculty of Medicine, Udayana University \\ ${ }^{2}$ Department of Geriatric Division of Internal Medicine Department \\ ${ }^{3}$ Department of Medical Education for International Cooperation, International Research Center for Medical Education, \\ Graduate School of Medicine, The University of Tokyo
}

\section{A R T I C L E I N F O}

\section{IAKMI IPHJI use only:}

Received date : 29 March 2020

Revised date : 17 April 2020

Accepted date : 20 May 2020

Keywords:

value based practice

elderly

interprofessional collaboration

\begin{abstract}
A B S T R A C T
Values-based practice is a new practice framework that promotes discussion focusing on the patient's and healthcare professionals' values for better clinical decision making. Our workshop was developed along with the framework of values-based practice. This workshop aimed to internalize diversity of values of both patients and health professionals and to rank interprofessional discussion as valuable to reach acceptable decision for both sides. The workshop involved healthcare professionals who worked in a public hospital in Bali. They were 6 medical doctors, 6 nurses, 4 nutritionist, 3 pharmacists, and 3 physiotherapists involved. The participants attended 1.5-hour lectures on comprehensive geriatric management and on interprofessional care for the elderly. In the next 1.5 hours the participants had simulated interprofessional conference for an elderly patient case. In the first half of the conference, participants formed groups of 5-6 people and played the roles of different healthcare members and a patient. The participants discussed the care management decision for the patient. In the latter half the participants started simulated discharge conference. After the conference, participants were asked to complete an evaluation questionnaire for this workshop. Twenty participants completed the questionnaire. Seven participants responded perfectly for the question "the workshop had internalized diversity of values of patients/healthcare professionals' sides" and the remaining responded fairly. Meanwhile $40 \%$ of participants ranked the interprofessional discussion as perfectly valuable to negotiate recognition between patient and healthcare side. Open-ended questions revealed that the discussion enhanced healthcare professionals' understanding of values of patients in delivering care for elderly patients. Interprofessional discussion allows internalization of values for both of patient and healthcare professionals' sides.
\end{abstract}

\section{INTRODUCTION*}

Value based practice (VBP) is a new practice framework in decision making in healthcare service delivery. This framework includes wider range of frameworks such as patient-centered medicine, biopsychosocial model, narrative-based medicine, open dialogue, clinical ethics, professionalism, and

\footnotetext{
* Corresponding author.

E-mail address: ketuternawati@umud.ac.id
}

interprofessional collaborative practice [1]. VBP is a methodology which focuses on promoting discussion of values between healthcare professionals and patients/carers. According to Oxford English Dictionary, value is the concept that something is held to deserve the importance, worth, or usefulness of something [2]. Cambridge English Dictionary also defines values as the beliefs people have especially about what is right and wrong and what is most important in life that control their 
behaviour. This means that VBP allow healthcare professionals to make clinical decision together with patient by taking into consideration their values as well as the values of the patient/carer. It is expected the decision made in accordance with what is right from both care providers and the patients as well. In VBP framework, partnership and collaboration between not only healthcare professionals who provide care to the patients is maintained but also with the patient as the expert or source of information of their illness/disease. VBP is considered as a complementary and support to evidence based practice for healthcare professionals in clinical decision making. VBP has four main skills to foster i.e. awareness, reasoning, knowledge and communication skills [3].

Skills of value awareness is very important to acknowledge something that we really need most yet we mostly do not realise it is there. It is just like the air we breath. We need the air to live but because it happens naturally, sometimes it needs our awareness of this air. Similar case to our awareness of values. Both healthcare professionals and patients need to be aware of the presence of values and taking into consideration of this value in delivering the care. The second is reasoning skills of value based practice. This reasoning skill aims to explore different values which may exist in different and complex health situation. In fostering this skills, healthcare professionals need to hold on to ethics principles i.e. autonomy, healthcare professionals should act to the benefits of their patient (beneficence), do no harm (non maleficence) and justice. Knowledge of value based practice can be gained from exploring patient's value from interviewing the patient, their families and even getting information from scientific resources using psychological approach. Last but not least skills is communication skills. This skill is required in dealing with patient and healthcare professionals. Although healthcare professionals shared the same value of treating the same patient, however, in communication skill, the healthcare professionals need to be aware of their own values in treating the patient. For instance, a medical doctor has concerns of the patient treatment, whereas the physiotherapist has concerns of making less harm to the patients. The differences of values amongst healthcare professionals needs to be revealed to reduce the risk of communication failure. Healthcare professionals may learn values awareness and communication skills through interprofessional collaborative practice.

In Indonesia, there is limited information regarding research on VBP and its awareness of patient's value through interprofessional collaborative practice. This study was a workshop of interprofessional conference as a means of collaboration involving different healthcare professionals in treating an elderly patient. Elderly patient is one group of population which may have complex health problems. A Swiss Cheese Model is one of the methods which may be employed in comprehensive assessment to the elderly. The model consists of eight dimensions such as social/caregiver, economic, environmental, nutritional, medical/surgical, rehabilitation, cognitive and emotional aspect [4]. The aimed of this study was to identify whether the workshop were educationally successful.

\section{METHOD}

The investigators contacted healthcare professionals who worked in geriatric departments in hospitals in Bali to attend the workshop. Objectives of the workshop were set as (1) participants will be able to internalize diversity of the values of both patient/healthcare sides, and (2) participants will be able to rank interprofessional discussion as valuable to negotiate recognition between patient and healthcare sides. The participants attended 1.5-hour lectures on comprehensive geriatric management and on interprofessional care for the elderly. In the next 1.5 hours the participants had simulated interprofessional conference for an elderly patient case. In the first half of the conference, participants formed groups of 5-6 people and played the roles of different healthcare members and a patient. The participants discussed the care management decision for the patient. In the latter half the participants started simulated discharge conference. After the conference, participants were asked to complete an evaluation questionnaire for this workshop. The questionnaire has ten questions to identify the participants perspective as to whether they reached the goals of the workshop and how to 
improve the workshop for improvement to foster value awareness both for the patient and the healthcare providers. Quantitative data was analysed descriptively from the response provided while the opened ended questions were analysed thematically.

\section{RESULTS AND DISCUSSION}

The number of participants in the workshop were 22, including six medical doctors, six nurses, four nutritionists, three pharmacists, and three physiotherapists. However, only 20 participants returned the evaluation sheet. The majority of participants was female and they have been working in the area of geriatric ranged from 3 to 15 years.

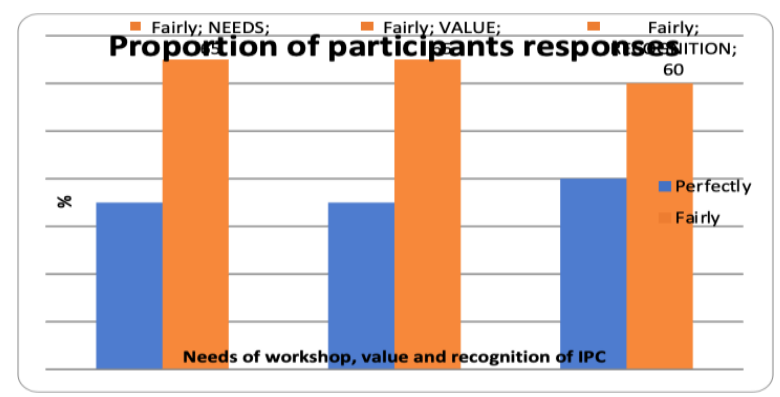

Fig 1. Proportion of participants responded to the needs of VBP workshop and value awareness and recognition of IPC to foster the awareness.

Figure 1 illustrates all participants responded that the workshop on interprofessional conference met their needs in clinical practice in which $35 \%$ of participants indicated perfectly matched their needs. The participants also responded that a workshop on interprofessionals conference internalised their diversity of values in clinical decision making. Further, forty percent of the participants recognised that interprofessional conference fasilitated their awareness of values diversity amongst healthcare professionals and the patients.

Some interesting responses to the workshop were shown in open ended questions and illustrated below.

"The workshop showed us how to take care of elderly patients in a very good way with problem solving from the perspectives of interdisciplinary professionals"
"We can decide the quality life of a patient with discussion with others"

"We have to include the values of a patient and family in clinical decision making" $\backslash$

"Interdisciplinary perspectives are important in treating elderly patient"

"interprofessional collaboration should be included within local and social context"

The VBP workshop we held was successful to achieve the objectives and to let participants understand as one of the methods in clinical decision making. Fulford highlighed the importance of VBP in psychiatric diseases in the beginning but later suggested implementation of VBP across various medical disciplines. Petrova et al [5] also emphasised that VBP allows better connection between the individual and evidence based practice.

Interprofessional collaborative practice provides collaboration amongst healthcare professionals. If the collaboration was initiated smoothly, it will foster positive collaboration competencies such as enhancing interprofessional communication skills, improving understanding of each other roles, as well as cultivating values of other healthcare professionals as well as the patients. Healthcare providers need experiences to work in a team thus they could reflect their learning to be fostered in their clinical practice. Cultivating collaboration between healthcare professionals is not an easy task. However, if healthcare professionals put the patient as their main value of their service, this would enhance the professionals value of respect of the patient before themselves. Thus, it is expected the healthcare professionals should provide a better clinical decision making by taking into consideration not only their values but also the patients' values as well.

Insufficient number of participants is one of limitations of the study. Although participants recruitment has been conducted well ahead before the workshop, some participants cancelled their participation due to unavoidable commitment in their work place.

\section{CONCLUSION}

This study found that interprofessional discussion enhanced internalising of values both of the healthcare professionals and the patients. The discussion in collaboration 
between healthcare professionals is valuable in clinical decision making. Future study is suggested to involved more participants in the workshop to provide a more valid result.

\section{ACKNOWLEDGMENT}

Researchers would like to thank Academic Affair of Medical Department Faculty of Medicine Udayana University for facilitating this workshop.

\section{REFERENCES}

[1] Jill Thistlewaite. (2012). Value based interprofessional collaborative practice: working together in healthcare. Cambridge University Press. https://doi.org/10.1017/СBO978113910890 4

[2] Value. (2019). In Oxford Online Dictionary. Retrieved from https://en.oxforddictionaries.com/defi nition/value

[3] Fulford K. W. (2008). Values-based practice: a new partner to evidence-based practice and a first for psychiatry?. Mens sana monographs, 6(1), 10-21. doi:10.4103/0973-1229.40565

[4] Bautista. M. \& Meuleman J. (2009) "Swiss Cheese Model" approach to clinical geriatrics - Geriatric Interdisciplinary Care Summary (GICS). POGOe - Portal of Geriatrics Online Education. Available from: https://pogoe.org/productid/20570

[5] Petrova, M., J., \& Fulford, B. K. (2006). Values-based practice in primary care: easing the tensions between individual values, ethical principles and best evidence. The British journal of general practice: the journal of the royal College of General Practitioners, 56(530), 703-709 